\title{
O mapeamento de processos como ferramenta para tomada de decisão na implantação de SIG: estudo de caso de uma lavanderia hospitalar
}

\author{
Gianni Lara \\ Assessora Técnica da AHMG, Conselheira Estadual de Saúde e \\ Conselheira Municipal de Saúde de BH. \\ nucleotecnico@ahmg.com.br
}

RESUMO: Objetiva-se conceituar SIG - Sistema de Informações Gerenciais e Mapeamento dos Processos, suas correlações e relevância para a gestão hospitalar. Apontar sugestões e um modelo de SIG para a Lavanderia do Hospital Y. O SIG é um instrumento administrativo que aperfeiçoa a comunicação, melhora o processo decisório pelo uso eficiente dos seus recursos, mas que, para isso, exige da organização um investimento relativamente alto em tecnologia da informação (Hardware e Software) e capacitação de pessoal. Ao levar em consideração os processos e mapeá-los, é possível conhecer e diagnosticar melhor as atividades desenvolvidas, conectar e difundir conhecimentos e potencializar o grau de sucesso na implantação do sistema. A metodologia adotada contemplou estudo de caso, pesquisa bibliográfica, pesquisa documental, observação participante e aplicação de entrevistas estruturadas junto aos empregados do setor. Concluiu-se que, ao conhecer melhor os processos que serão controlados pelo SIG, potencializa-se o grau de sucesso de sua implantação e que, a partir de uma visão integrada dos processos, os serviços de informação podem atingir seus objetivos de forma mais satisfatória.

PALAVRAS CHAVES: sistema de informação gerencial, mapeamento de processos, lavanderia hospitalar, administração hospitalar.

\section{INTRODUÇÃO}

O Hospital Y é um hospital filantrópico considerado de referência microrregional para a região do Campo das Vertentes. Optou-se por desenvolver um trabalho de pesquisa no setor de lavanderia desta Instituição devido ao fato de que este estava em fase de implantação de um SIG. Segundo o Administrador do Hospital Y, esta implantação poderia possibilitar: qualidade no atendimento, melhor controle do enxoval, apuração eficaz dos custos e melhor produtividade.

Durante 65 (sessenta e cinco) horas foram acompanhados todos os processos para lavagem das roupas hospitalares. A intenção não foi dar a solução definitiva para a implantação do SIG na lavanderia, mas sugerir algumas ações que poderiam ser viáveis, funcionais e eficientes para atingir os objetivos gerenciais do Hospital Y e assim contribuir com esta entidade tão importante para a sua região.

\subsection{Contextualização}

O setor de prestação de serviços vem se destacando na economia mundial, ao mesmo tempo também está sendo muito cobrado pelos seus clientes. Santos et al. (2003) afirmam que a busca de melhoria da qualidade, tão comum em empresas industriais, passou a ser prioridade também nas organizações prestadoras de serviços.

Para tal, muitas empresas têm maciçamente investido em Tecnologia da Informação (TI) com o intuito de buscar uma maior eficácia dos seus processos operacionais e decisórios, melhorando a qualidade dos seus produtos e/ou serviços, além de satisfazerem as necessidades dos seus clientes cada vez mais exigentes.

Para Prates \& Ospina (2004) os administradores na sua maioria investem em novas ferramentas de tecnologia da informação, porque acreditam que isso lhes permitirá realizar suas operações de forma mais rápida a um custo mais baixo.

O nome do Hospital foi modificado visando à privacidade dos dados. 
Identifica-se também nas empresas, segundo Moreira (2003), no que se refere à tomada de decisões, que os executivos precisam ter mais disponibilidade de informações e menos dados, afinal, a única informação correta é aquela entregue na hora certa à pessoa certa.

A forma como a informação é obtida, organizada, gravada, recuperada e utilizada permite ao gerente atuar com mais segurança, aumentando a possibilidade de acerto na tomada de decisão (GUIMARÃES 2004).

Segundo Telles Filho \& Cassiani (1999), o conhecimento científico e tecnológico é atualmente um processo em acelerado desenvolvimento. O computador como principal instrumento da informática auxilia na organização do elevado volume de informações da atual sociedade.

A utilização da informática na área da saúde no Brasil apresenta um atraso considerável quando comparado a outros países. Entretanto, a partir da década de 80 observou-se um avanço importante nessa área. A utilização do computador iniciou-se na área hospitalar administrativa para registro da admissão do cliente, busca de leitos, internação e estudos estatísticos. (GUIMARÃES 1988 apud GALVAO; SAWADA 1996).

A Informática tem proporcionado grandes contribuições para a área da saúde, embora ainda existam muitos desafios a serem superados. A organização de saúde é um ambiente que instiga à informatização. A informatização hospitalar hoje é uma prioridade para evitar falhas e ter maior controle sobre as informações. Desta forma, para a criação desses ambientes informatizados é necessário escolher o melhor programa, integrá-lo ao equipamento adequado, qual base de dados utilizar, além da arquitetura de rede a ser usada. Tudo isso exige um apanhado abrangente de conhecimento profissional (BIANCHINI et al. 2002).

Nos atuais serviços de saúde, os bons cuidados do paciente e os melhores resultados são vistos como "produto" das ações e interações de todas as pessoas envolvidas no processo. A performance é assim, o reflexo de uma grande variedade de sistemas e subsistemas que congregam as funções essenciais diárias (MEZOMO, 1995 apud BARTOLOMEU 1998).

A informação é um recurso disponível e democrático na atualidade. Contudo, os sistemas de informação são implantados nas empresas sem conhecimento técnico adequado para atingir os efeitos positivos almejados, o que pode ser atribuído a uma questão de desvio de foco, pois muitas empresas não definem claramente para que serão usados os equipamentos e sistemas antes de comprá-los. Máquinas e softwares nada resolvem se não forem absolutamente apropriados para uma finalidade específica (D'ERCOLE 1998 apud BERALDI; ESCRIVÃO FILHO 2000).

Segundo Schenatz (2005) todas as fases de desenvolvimento e implantação de um Sistema de Informações Gerenciais - SIG devem ser debatidas pelo gerente que, acima de tudo, necessita conhecer profundamente a estrutura da organização. Antes de desenvolver e operacionalizar um SIG, o administrador precisa identificar as finalidades para as quais o SIG foi ou deve ser estruturado, o que evita o desenvolvimento de sistemas que forneçam dados e informações irrelevantes para o processo decisório da organização.

\subsection{Objetivos do estudo}

O trabalho teve como objetivo geral:

- Demonstrar a relevância do Mapeamento de Processos para a elaboração e implantação de um Sistema de Informações Gerenciais (SIG).

- Como objetivos específicos para a execução do objetivo geral, foram definidos:

- Conceituar Sistema de Informações Gerenciais e Mapeamento dos Processos;

- Conhecer o impacto da relação entre Mapeamento de Processos e Sistema de Informações Gerenciais (SIG)

- Apontar algumas sugestões e um modelo simples de SIG para melhoria do controle do enxoval na Lavanderia da Instituição em questão.

\subsection{Justificativa e relevância do tema}

SIG é um instrumento administrativo que aperfeiçoa a comunicação, melhora o processo decisório pelo uso eficiente dos seus recursos, mas que para isso exige da organização um investimento relativamente alto em tecnologia da informação (Hardware e Software) e capacitação de pessoal.

Assim, o trabalho teve como propósito servir de norteador aos hospitais que pretendam implementar os seus SIG. Ao levar em consideração os processos e mapeá-los é possível diagnosticar melhor as atividades desenvolvidas, homogeneizar conhecimentos e potencializar o grau de sucesso na escolha e adoção desses sistemas. Também dependendo do porte do hospital o sistema pode ser mais simplificado e econômico sem deixar de ser eficiente, afinal é o sistema que tem que se adaptar aos processos e não o contrário. 


\subsection{Metodologia}

Foram adotados os seguintes critérios de Pesquisa Social de Gil (1999): um estudo de caso realizado na Lavanderia do Hospital "Y", pesquisa bibliográfica, pesquisa documental de segunda mão - que de alguma forma já receberam algum tipo de análise como relatórios e tabelas estatísticas da empresa estudada, observação participante artificial e aplicação de entrevistas estruturadas junto aos empregados do setor conforme modelo em apêndice.

Desta forma pretendeu-se minimizar as limitações inerentes a cada um dos métodos utilizados.

\section{SISTEMA DE INFORMAÇÃO GERENCIAL}

Algumas definições e considerações sobre SIG segundo autores:

Para Moreira (2003), o Sistema de Informações Gerenciais é um instrumento administrativo que pode otimizar as comunicações e o processo decisório nas empresas, que tem como propósito básico de habilitar a empresa a alcançar seus objetivos pelo uso eficiente dos recursos disponíveis (pessoas, materiais, equipamentos, tecnologia, dinheiro e informação).

"Sistema de Informação Gerencial é o conjunto de tecnologias que disponibiliza os meios necessários à operação do processo decisório em qualquer organização por meio do processamento dos dados disponíveis" (CRUZ 1998:48 apud SCHENATZ 2005).

De acordo com Oliveira (2002) apud Schenatz (2005), SIG é o processo de transformação de dados em informações que são utilizadas na estrutura decisória da organização, bem como proporcionam a sustentação administrativa para otimizazação dos resultados esperados.

Contudo, Bufoni (2003) afirma que recentes estudos indicam existir um paradoxo nos sistemas de informação considerados teoricamente perfeitos; muitos se tornam um fracasso durante sua implementação, sendo as principais causas do sucesso e do insucesso dos sistemas de informações gerenciais atribuídas a quatro fatores: a complexidade, a participação do usuário, o apoio da direção e a equipe de desenvolvimento.

Muitas publicações de fracassos na implantação de sistemas de informação devido aos altos investimentos e baixa produtividade têm trazido cada vez mais pontos à discussão (BALLANTINE et al. 1996 apud BUFONI 2003).

Desta forma, os quatro fatores, sugeridos por Bufoni (2003) como principais causas do sucesso e do insucesso na implantação de um SIG devem ser atentamente trabalhados e correlacionados.

Afinal, uma dada tecnologia não é automaticamente boa ou má. Seu resultado dependerá da maneira como esta tecnologia será aplicada. O aumento da precisão organizacional, auxiliada por sistemas de informação, trará maior eficiência na administração de seus processos, recursos e atividades e maior eficácia na obtenção de resultados previamente estabelecidos (SOLOMON 1986 apud PRATES, OSPINA 2004).

Para Oliveira (2002) apud Schenatz (2005), o SIG é composto, de uma forma abrangente, pelos seguintes elementos: 
Figura 1 - Componentes do SIG

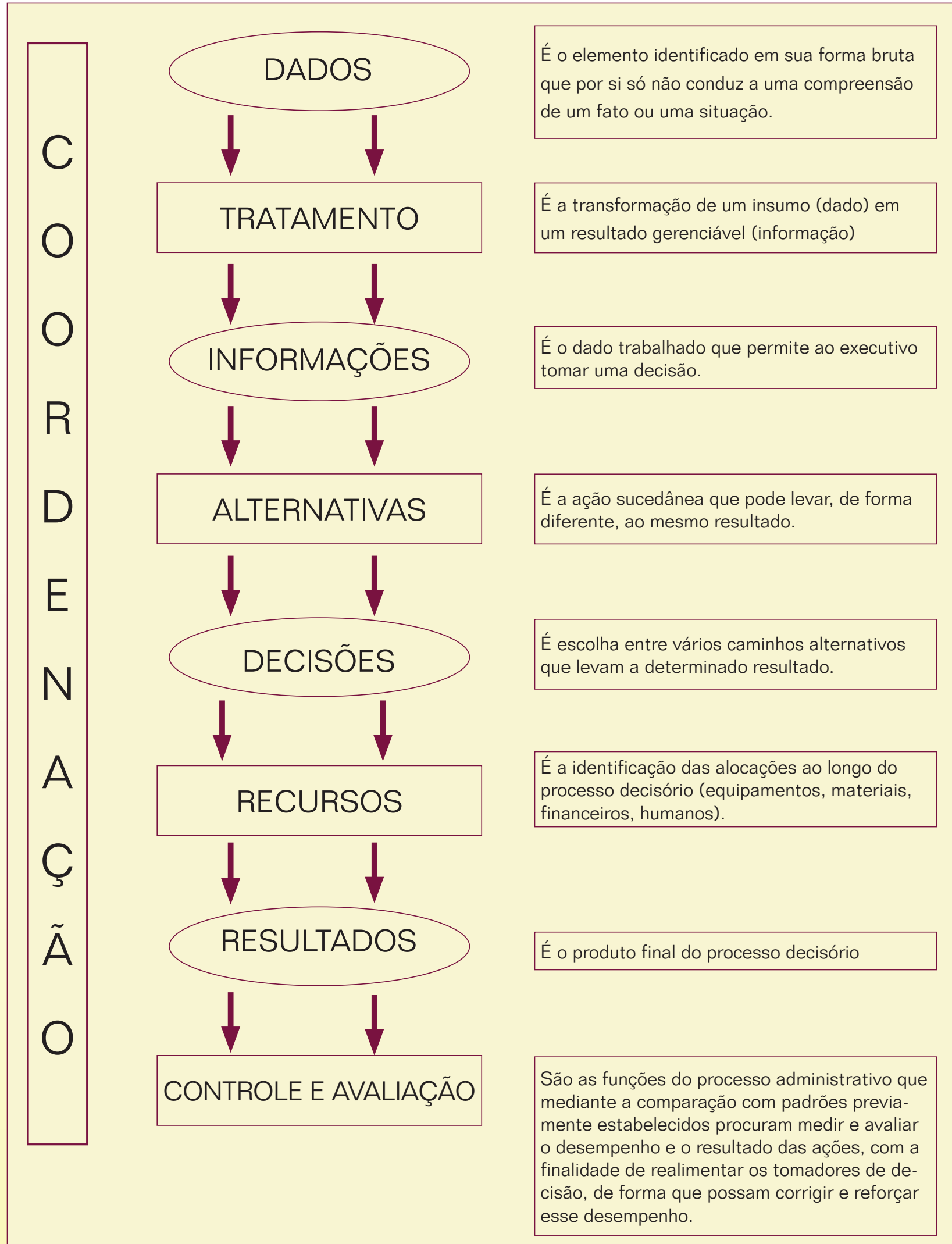

Fonte: SCHENATZ (2005:26) 


\section{MAPEAMENTO DOS PROCESSOS}

Conceitualmente, processo é a palavra originária do Latim processu, significa "Ato de proceder, de ir por diante, maneira pela qual se realiza uma operação, segundo determinadas normas, método, técnica" (FERREIRA, 1986:1395 apud SENTANIN, 2004)

Processo é o conjunto de recursos e atividades inter-relacionadas que transformam insumos (entradas) em produtos (saídas). Essa transformação deve agregar valor na percepção dos clientes e exige certo conjunto de recursos [...] O processo poderá exigir a documentação da seqüência de etapas por meio de especificações, procedimentos e instruções de trabalho, bem como a definição das etapas de medição e controle. (IPEG 2005 apud DAMIANO; SAAD 2006)

Segundo a NBR ISO 9000 (2000:10) apud Valls (2004), processo é o "conjunto de atividades interrelacionadas ou interativas que transformam insumos (entradas) em produtos (saídas)"

Harrington (1993:41) apud Oliveira (2003) conceitua processo como sendo um grupo de tarefas interligadas logicamente, que utilizam os recursos da organização para gerar resultados definidos, de forma a apoiar os seus objetivos.

Ainda para Vilella (2000:12) apud Oliveira (2003), "um processo dispõe de inputs, outputs, tempo, espaço, ordenação, objetivos e valores que, interligados logicamente, irão resultar em uma estrutura para fornecer produtos ou serviços ao cliente."

Mapeamento de processos é um mecanismo para retratar a situação atual e descrever a visão futura dos processos de negócios (ERNEST, YOUNG, 1997 apud KINTSCHNER, BRESCIANI FILHO 2004).

Para Kintschner \& Bresciani Filho (2004) os principais objetivos do mapeamento de processos são garantir:

- melhoria dos processos, tendo como objetivo eliminar processos e regras obsoletas e ineficientes e gerenciamento desnecessário;

- padronização de documentação;

- facilidade na documentação;

- destreza de leitura;

- homogeneidade de conhecimento para todos os membros da equipe;

complemento total na documentação dos processos.

Ao mapear os processos, o administrador pode entender e diagnosticar melhor quais são as atividades executadas e desenvolvidas em um determinado setor, a fim de compreender os verdadeiros propósitos da adoção de um Sistema de Informação Gerencial e aumentando o percentual de acerto na escolha do mesmo.

Como aponta Lozinsky (1996) apud Oliveira (2003), a implementação de um pacote de software significa a substituição de alguns sistemas e processos atuais, e a adaptação de certos procedimentos e controles. Razão esta, que é preciso analisar como estão funcionando esses sistemas e processos atuais.

\section{ESTUDO DE CASO}

A lavanderia hospitalar é um dos serviços de apoio ao atendimento dos pacientes, responsável pelo processamento da roupa e sua distribuição em perfeitas condições de higiene e conservação, em quantidade adequada a todas às unidades do hospital (MINISTÉRIO DA SAÚDE, 1986).

As roupas hospitalares diferem daquelas utilizadas em outros tipos de instituições ou residências porque alguns itens apresentam-se contaminados com sangue, secreções ou excreções de pacientes em maior quantidade de contaminação e volume de roupa, mas não há distinção das sujidades encontradas nas roupas da comunidade em geral (SECRETARIA DA CASA CIVIL, 2006).

A operacionalização da lavanderia abrange todo o circuito da roupa, desde a sua utilização nas unidades do hospital, passando pela coleta da roupa suja nessas unidades, até sua redistribuição após o devido processamento. A utilização correta da roupa contribui para a conservação dos tecidos, para o conforto do usuário e para a redução dos gastos. 


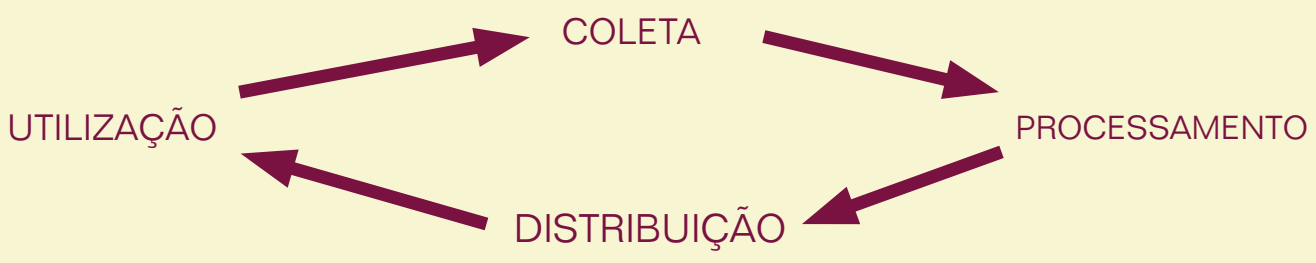

Fonte: MINISTÉRIO DA SAÚDE (1986)

\subsection{Mapeamento dos processos da lavanderia do hospital " $Y$ "}

A lavanderia hospitalar estudada é dividida nas seguintes áreas:

- Área recepção (separação, pesagem e lavagem);

- Área acabamento

- molhada (centrifugação);

- seca (secagem, calandragem e plastificação);

- rouparia (guarda descanso e distribuição);

- Sala de Costura.

Figura 3: Fluxo funcional da lavanderia do Hospital $Y$

\section{ÁREA DE RECEPÇÃO}

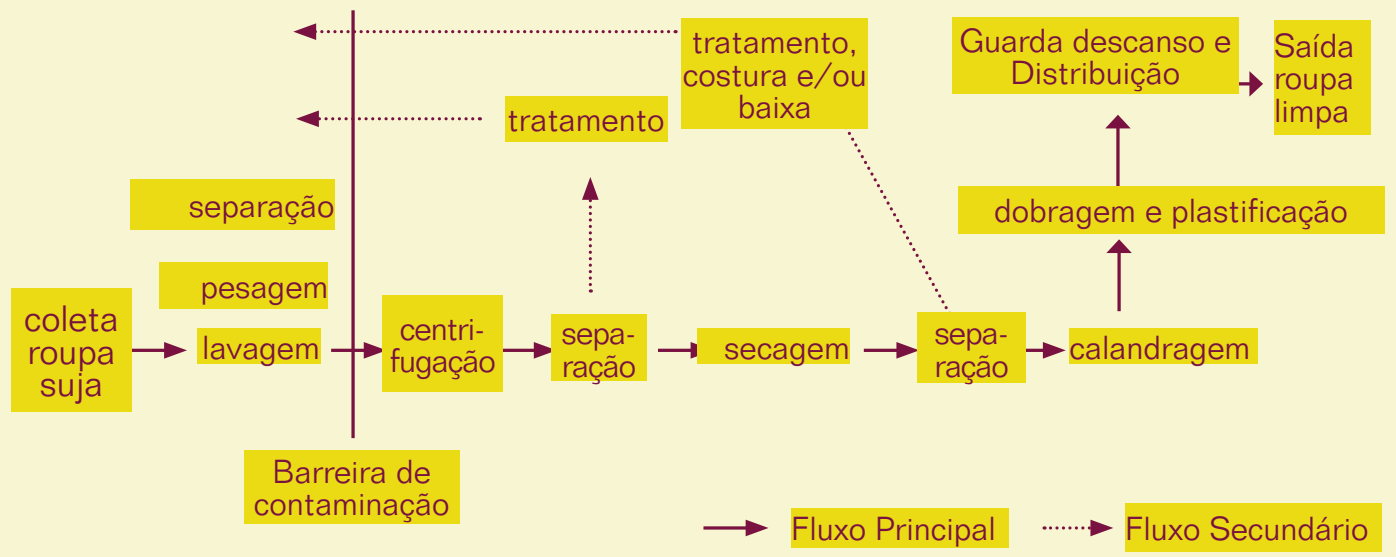

O processo de trabalho na lavanderia inicia-se com a:

- Coleta da roupa suja - é realizada nas unidades hospitalares, em horários pré-estabelecidos. A roupa suja é conduzida em um carrinho próprio com tampa até a área de recepção.

- Separação - a roupa é classificada de acordo com o grau de sujidade e tipo de tecido. Essa classificação tem a finalidade de agrupar a roupa que pode ser lavada em conjunto e a que terá o mesmo acabamento. Na separação, é indispensável observar e retirar algum instrumento que por ventura tenham sido encontrados meio as roupas como: instrumentos perfuro cortantes, sacos plásticos, luvas de procedimentos e outros objetos. Desta forma evita-se que estes elementos estranhos entrem no processo de lavagem, causando danos às máquinas e ao processo além de possíveis acidentes de trabalho.

- Pesagem - após a separação, a roupa é pesada e agrupada em fardos correspondentes a uma fração da capacidade da máquina, em geral $80 \%$ de sua capacidade de lavagem, além de ser identificado quanto ao tipo de processamento a que deverá ser submetido em função do tipo de sujidade. A pesagem da roupa é indispensável para indicar a carga correta das lavadoras, o peso da roupa recebida de cada unidade para a contabilidade de custos. 
- Lavagem - é o processo que consiste na eliminação da sujeira fixada na roupa e redução do nível bacteriológico deixando a roupa em condições de uso. O processo de lavagem é realizado na área recepção, o ciclo a ser empregado no processo de lavagem é determinado de acordo com o grau de sujidade, tipo da roupa, tipo de equipamento da lavanderia e dos produtos utilizados.

- Centrifugação - as máquinas de lavar possuem barreiras, ou seja, com duas portas de acesso, uma para cada área, assim a roupa pode ser retirada, após o processo de lavagem no lado da área de acabamento sem haver mais contato com a área de recepção separadas por meio de uma parede até o teto (barreira de contaminação) evitando assim a dispersão dos microrganismos pelas áreas de acabamentos, o que levaria à recontaminação da roupa. A barreira é dotada de visores, para facilitar a comunicação e o controle. As roupas são retiradas da máquina de lavar pelo lado da área de acabamento e receberão a centrifugação para retirar o excesso de água.

Separação - terminada a centrifugação a roupa é retirada e selecionada, quando a roupa não apresentar um grau de limpeza aceitável, recebendo tratamento especial dependendo do tipo de sujidade presente no tecido e retornando a área de recepção para ser novamente lavada. As demais roupas são encaminhadas para a secagem.

- Secagem - depois de secar, as roupas são retiradas da secadora e colocadas em mesas apropriadas para dobragem ou para calandragem. Durante esse procedimento, ainda é possível encontrar roupas que precisam de tratamento e/ou peças danificadas que vão ser separadas e encaminhadas ao setor de costura para consertado ou baixa.

- Calandragem - É a operação que seca e passa ao mesmo tempo as pecas de roupa lisa (lençóis, colchas leves, campos, etc.). Geralmente são necessários dois operadores para colocar a roupa na calandra, e um para retirar e dobrar a roupa seca e passada.

- Plastificação - as peças são unitariamente colocadas e lacradas em sacos plásticos, evitando assim possíveis contaminações durante o período de guarda descanso (armários onde ficam as roupas até o momento de serem distribuídas) e/ou durante o processo de distribuição nas unidades hospitalares.

A lavanderia possui: 4 máquinas de lavar (3 máquinas de 50 kg e 1 máquina de 15kg), 2 centrifugas, 3 secadoras, 1 calandra, 1 máquina para lacrar as peças plastificadas e 2 máquinas de costuras.

A lavanderia do Hospital Z funciona todos os dias das 6:30 às 19:00. O processo de lavagem da roupa, para uma máquina de lavar de $50 \mathrm{~kg}$, varia entre 50 minutos à 1 hora e 30 minutos dependendo do ciclo de lavagem. O enxoval é marcado com tinta de tecido com as siglas dos setores a que pertence o que facilita o controle das peças.

A Lavanderia conta com um quadro de pessoal com 13 empregados, sendo que 11 empregados trabalham com escalas de trabalho de 12 por 36. Distribuídos nos setores da seguinte forma: 1 empregado na área de recepção (fazendo a coleta e lavagem da roupa), 4 empregados na área de acabamento. A Costureira e a Coordenadora do setor são diaristas. O esquema de rodízio é adotado nas atividades executadas, todos são capazes de operar todas as máquinas da lavanderia.

Foi realizada uma entrevista estruturada em 11 dos 13 empregados da lavanderia perfazendo um total de $85 \%$ (oitenta e cinco por cento) do número de empregados. Analisou-se com as entrevistas: o perfil desse empregados, a forma de execução das suas rotinas de trabalho e colhidas sugestões de como melhorar o controle do enxoval.

\section{ANÁLISE DOS RESULTADOS}

Dos $85 \%$ dos empregados entrevistados, 18\% possuem até 30 anos de idade, $18 \%$ de 31 a 40 anos, 55\% possuem entre 41 a 50 anos e $9 \%$ tem mais de 50 anos. No que diz respeito ao sexo, 55\% são mulheres e $45 \%$ homens. Apesar de todos os empregados saberem operar todas as máquinas da lavanderia, os homens são os operadores responsáveis pelas máquinas pesadas - máquina de lavar, centrifuga e secadora, porque essas máquinas exigem um esforço físico maior. 
Tabela 1: Distribuição dos empregados segundo grau de escolaridade.

\begin{tabular}{lc}
\hline Grau de Escolaridade & $\%$ \\
\hline Sem Instrução & 9,09 \\
$1^{\circ}$ grau incompleto & 36,36 \\
$1^{\circ}$ grau completo & 9,09 \\
$2^{\circ}$ grau incompleto & 9,09 \\
$2^{\circ}$ grau completo & 36,36 \\
Superior incompleto & 0,00 \\
Superior completo & 0,00 \\
\hline \multicolumn{1}{c}{ TOTAL } & 100,00 \\
\hline
\end{tabular}

Cabe ressaltar que o fato de 64\% dos empregados terem mais de 41 anos e que praticamente 55\% deles não chegaram aos segundo grau de escolaridade dificulta bastante a implantação de um sistema de informação mais complexo e sofisticado, porque despenderia mais recurso para treinar esse pessoal, sendo que foi constatado que apenas um empregado tem familiaridade com uso do computador.

Tabela 2: Distribuição dos empregados segundo tempo de trabalho na Instituição.

\begin{tabular}{lc}
\hline Tempo de Trabalho na Instituição & $\%$ \\
\hline de 1 a 5 anos & 27,27 \\
de 6 a 10 anos & 18,18 \\
de 11 a 15 anos & 18,18 \\
mais de 15 anos & TOTAL \\
\hline
\end{tabular}

O tempo de trabalho na Instituição também se faz um agravante porque quase 55\% dos empregados estão na Instituição a mais de 10 anos, ou seja, com uma cultura organizacional muito arraigada e com alta resistência as mudanças. E quando questionados com quem haviam aprendido a forma de executar suas tarefas: com um técnico de lavanderia, com a coordenação do setor ou com um colega de trabalho mais antigo, categoricamente $100 \%$ afirmaram que foi com um colega de trabalho. Contudo, a Instituição tem a assessoria periódica de um técnico de lavanderia que é o seu próprio fornecedor de produtos químicos. A Entidade também não dispunha de um setor de Recursos Humanos que seria o principal responsável pela contratação e treinamento de pessoal.

Indagou-se aos empregados, com base em suas experiências, quais seriam os principais motivos da perda do enxoval, $45 \%$ deles acreditam que as baixas por tempo de uso são as principais causas da redução do enxoval, pois não há uma reposição das peças na mesma proporção, o que acontece devido às limitações financeiras. O Manual de Lavanderia Hospitalar do Ministério da Saúde define que a quantidade de roupa necessária no hospital varia de 4 a 6 mudas, sendo uma no leito; uma a duas a caminho da lavanderia em fase de processamento; uma a duas prontas, "em descanso"; meia a uma muda na rouparia da unidade. A Instituição estudada vinha operacionalizando em média com 2 a 3 mudas.

Também o sumiço de peças é apontado por 36\% dos empregados como um dos maiores responsável pela redução do enxoval. Assim, a limitação dos recursos financeiros associada ao desaparecimento de peças aponta a necessidade da implantação de um Sistema de Informação Gerencial que possa monitorar mais efetivamente esse enxoval.

\section{PROPOSTA DE SIG PARA LAVANDERIA DO HOSPITAL "Y"}

Com todas essas informações levantadas através de um mapeamento dos processos de trabalho na lavanderia, não seria recomendado investir num SIG muito sofisticado porque os empregados não estariam aptos a operacionalizá-lo, o investimento nesse tipo de treinamento não é barato e as limitações financeiras são grandes. O que poderia acarretar um desperdício de recursos, já que o sistema não seria devidamente aproveitado e os objetivos desejados comprometidos. 
Torna-se necessário então pensar num sistema de informação gerencial mais simples que possa ser efetivamente operacionalizado, sem deixar de ser eficaz proporcionando os resultados almejados.

A roupa suja hospitalar deve ser minimamente manuseada, para evitar contaminações, assim o controle de entrada das peças na lavanderia fica comprometido, sendo possível apenas contabiliza-las no final do processo de lavagem. A Instituição já possui um inventário do enxoval separado por setor. Sugeri-se, então, que os empregados da lavanderia na hora de entregar as roupas aos setores, preencham uma planilha relacionando o tipo de artigo com o setor a que a roupa está sendo entregue, utilizando para isso uma marcação por freqüências agrupadas de cinco em cinco.

\section{Exemplo:}

A coordenação do setor ou alguém designada por ela deveria supervisionar se as anotações de saída estão sendo feitas corretamente, anotar as peças que deram entradas e não saíram da lavanderia, acompanhar de perto o processamento da roupa para verificar se as peças não estão sendo extraviadas durante a lavagem, já que não é recomendado a contagem das mesmas antes do processo.

A lavanderia já possui um computador disponível e a coordenadora já recebeu treinamento básico de informática, então com o auxilio do programa Microsoft Access, uma boa ferramenta de controle de banco de dados, é possível criar e gerenciar tabelas de dados, consultas relacionadas, filtros, formulários para entrada e saída de dados, relatórios diversos para impressão, até mesmo páginas web para divulgações em rede, sem precisar partir para uma solução de terceiros no que se refere a software empresarial. Além disso, a Instituição também possui um departamento de informática que poderia ficar a cargo do suporte técnico. A coordenadora assim, alimentaria o banco de dados para gerar os vários tipos de consultas e relatórios que a administração precisasse de forma simples, rápida e eficiente.

No final de uma semana, as saídas deveriam ser consolidadas e comparadas aos números do inventário e das peças que ainda permanecem na área de guarda descanso, verificando assim as peças que ainda não retornaram dos respectivos setores. Como o número de mudas de roupas é restrito fica mais fácil controlar porque o enxoval é praticamente lavado todos os dias e quase não tem tempo de descanso. Esse descanso é recomendado para prolongar a vida útil do enxoval.

Também seria valida uma visita periódica nos setores para vistoriar os armários das unidades no intuito de detectar peças que saíram da lavanderia, mas que ainda não foram utilizadas pelos setores.

\section{SUGESTÕES}

Algumas sugestões foram levantadas pelos próprios empregados para tentar reduzir a perda do enxoval são elas:

- Controlar a entrada de bolsas dos visitantes.

- Utilizar lençóis descartáveis no transporte dos defuntos para o necrotério.

- Manter contato com os hospitais que recebem os pacientes transferidos do Hospital Y para que a peça que por ventura for encontrada com o paciente seja posteriormente devolvida.

O enxoval do Hospital $Y$ não se difere em nada dos enxovais comuns, assim sugeri-se que à medida que a Instituição for repondo o seu enxoval, ela opte por um enxoval personalizado, com o nome da Instituição estampado em toda sua extensão. Uma ação que pode dificultar os extravios.

Proporcionar aos empregados do setor e corpo de enfermagem aulas de educação continuada como forma de orientação dos principais cuidados que se deve ter com o enxoval hospitalar e da importância de manter o enxoval em bom estado de conservação.

Capacitar mais empregados da lavanderia para operacionalização de computadores, criando um grupo de profissionais mais qualificados que atenderão no futuro a pretensão da direção do Hospital Y de montar um Sistema Integrado de Gestão. 


\section{CONSIDERAÇÕES}

Ao conhecer melhor os processos que se deseja controlar através de um SIG, o administrador potencializa o grau de sucesso na implantação do mesmo. E a partir de uma visão integrada dos processos implantados, os serviços de informação podem atingir seus objetivos de forma satisfatória, principalmente se a organização possuir uma política de qualidade definida e implantada. Independentemente desse fato, cabe aos gestores modernizar as práticas gerenciais de seus serviços, equacionando suas intenções de investimento a realidade dos seus processos. É necessário conhecer a fundamentação teórica e analisar os serviços de informação, visando extrair os melhores resultados possíveis.

Como não existe nenhum SIG perfeito e que atenda todas as necessidades de controle organizacional, vale salientar que uma boa contratação de pessoal associada a treinamentos continuados é uma boa forma de garantir o comprometimento dos empregados com ele mesmo e com a instituição.

\section{REFERÊNCIAS}

BARTOLOMEU, A. T.; Identificação e avaliação dos principais fatores que determinam a qualidade de uma lavanderia hospitalar. Tese de Mestrado Engenharia da Produção. UFSC. Florianópolis - Santa Catarina, 1998. Disponível em: http://teses.eps.ufsc.br/defesa/pdf/1672.pdf. Acesso: 24/11/2006.

BERALDI, L. C.; ESCRIVAO FILHO E. Impacto da tecnologia de informação na gestão de pequenas empresas. Ciência da Informação, Brasília, v. 29, n. 1, 2000. Disponível em: <http://www.scielo. $\mathrm{br} /$ scielo.php?script $=$ sci_arttext\&pid $=$ S010019652000000100005\&lng $=p t \& n r m=i s o>$. Acesso em: 01 Nov 2006.

BIANCHINI, J. R. L.; POZZEBON, E.; ALMEIDA, M. A. F. Sistema de Informação Hospitalar no Planalto Serrano Catarinense. In: XXII Congresso da SBC. XXIX Seminário Integrado de Sofware e Hardware, 2002, Florianópolis. Disponível em: <www.anpad.org.br/rac/rac_indice_vol_07_n_04.html>. Acesso em: outubro de 2006.

BUFONI, A. L.; Uma análise dos fatores críticos de sucesso e insucesso na implementação de sistemas de informação gerencial: estudo do caso do segmento de exploração e produção de petróleo da Petrobras S.A. Tese de Mestrado em Ciências Contábeis - UFRJ. Rio de Janeiro, 2003. Disponível em: <http://www.bufoni.com/publica/tese.PDF>. Acesso em 21 Dez 2006.

DAMIANO L. C. C., SAAD M. R. M. O Mapeamento dos Processos da Dibd/Esalq: Assegurando d Qualidade através da Especificação dos Produtos. In: XIV SNBU - Seminário Nacional de Bibliotecas Universitárias, 2006, Salvador. Disponível em: <http://www.snbu2006.ufba.br/soac/ viewpaper.php?id=409>. Acesso em: 06 Nov. 2006.

GALVAO, Cristina Maria; SAWADA, Namie Okino. The use of computer technology in basic and hospital networks in the city of Ribeirão Preto (Brazil). Rev. Latino-Am. Enfermagem. Ribeirão Preto, v. 4, spe, 1996. Available from: <http://www.scielo.br/scielo.php?script=sci_arttext\&pid=S010411691996000700006\&lng=en\&nrm=iso >. Access on: 20 Jan 2007. doi: 10.1590/S010411691996000700006.

GIL, A. C.; Métodos e técnicas de pesquisa social. 5. ed. São Paulo: Atlas, 1999.

GUIMARÃESS, E. M. P.; Sistema de informação hospitalar: instrumento para tomada de decisão no exercício da gerência de unidades funcionais. Tese de Doutorado. Escola de Enfermagem de Ribeirão Preto USP, 2004. Disponível em: <http://www.teses.usp.br/teses/disponiveis/22/22132/tde-20032006095010/> Acessado: em 26/08/06.

MINISTÉRIO DA SAÚDE. Manual de Lavanderia hospitalar. Brasília, 1986. Disponível em: <bvsms. saude.gov.br/bvs/publicacoes/lavanderia.pdf>. Acesso em: 16/02/2007

MOREIRA, V. J.; Avaliação da estrutura do sistema de informações gerenciais nas empresas da indústria de pesca da foz do rio itajaí-açú. Tese de Mestrado em Engenharia da Produção da UFSC. Florianópolis, 2003. Disponível em: <http://teses.eps.ufsc.br/defesa/pdf/8577.pdf>. Acesso em: 02 Fev. 2007.

OLIVEIRA, N. M.; Seleção de sistemas de gestão e o impacto no processo de implantação: um estudo de casos múltiplos. Monografia Bacharel em Informática. Universidade do Vale do Rio dos Sinos - Unisinos. São Leopoldo, Rio Grande do Sul - RS, 2003. Disponível em: <http://inf.unisinos.br/ alunos/ arquivos/NatachaOliveira.pdf $>$. Acesso em: 22/01/2007. 
PRATES, G. A., OSPINA, M. T. Tecnologia da Informação em Pequenas Empresas: Fatores de Êxito, Restrições e Benefícios. RAC $\square$ Revista de Administração Contemporânea, v. 8, n. 2, Abr./Jun. 2004. Disponível em: <http://www.anpad.org.br/rac/vol_08/dwn/rac-v8-n2-gap.pdf >. Acesso em: 03 Nov 2006.

SANTOS, Luciano Costa; FACHIN, Gleisy Regina Bóries; VARVAKIS, Gregorio. Managing service processes in libraries. Ci. Inf., Brasília, v. 32, n. 2, 2003. Disponible en: <http://www.scielo.br/ scielo.php?script $=$ sci_arttext\&pid=S0100-19652003000200010\&Ing $=$ es\&nrm $=i s o>$. Acceso el: 20 Ene 2007. doi: 10.1590/S0100-19652003000200010.

SCHENATZ, B. N.; Utilização de data mining em um sistema de informação gerencial para o diagnóstico da formação de professores da graduação. Tese de Mestrado em Engenharia da Produção da UFSC. Florianópolis, 2005. Disponível em: <http://teses.eps.ufsc.br/defesa/pdf/8577.pdf > . Acesso em: 02 Fev. 2007.

SECRETARIA DA CASA CIVIL. Prestação de Serviços de Lavanderia Hospitalar. São Paulo, 2006. Disponível em : <http://www.cadterc.sp.gov.br/Novembro-2006/VOL-10-Lavanderia\%20Hospitalar. pdf > . Acesso: 24/02/2007.

SENTANIN, O. F.; Gestão por processos em uma empresa de pesquisa e desenvolvimento: objetivo estratégico de um modelo de gestão. Tese de Mestrado em Engenharia da Produção. Escola de Engenharia de São Carlos da Universidade de São Paulo. São Carlos - SP, 2004. Disponibilizado em: <http://www.teses.usp.br/teses/disponiveis/18/18140/tde-01022005-085937/>. Acesso em 15/02/2007.

TELLES FILHO, Paulo Celso Prado; CASSIANI, Silvia Helena De Bortoli. The computer in the teaching of nursing: analysys of the attitudes by students from university institutions. Rev. Latino-Am. Enfermagem., Ribeirão Preto, v. 7, n. 1, 1999. Available from: <http://www.scielo.br/scielo. php?script $=$ sci_arttext\&pid $=$ S0104-11691999000100012\&lng $=$ en\&nrm $=$ iso $>$. Access on: 20 Jan 2007. doi: 10.1590/S0104-11691999000100012.

VALLS, Valéria Martin. Approach by NBR ISO 9001 processes applied to information services. Ci. Inf., Brasília, v. 33, n. 2, 2004. Available from: <http://www.scielo.br/scielo.php?script=sci_ arttext\&pid=S0100-19652004000200018\&lng=en\&nrm=iso >. Access on: 21 Jan 2007 . doi: 10.1590/S0100-19652004000200018.

KINTSCHNER, F. E.; BRESCIANI FILHO, E.; Reengenharia de processos: Transformando as necessidades do cliente em parâmetros de um sistema. Revista de Administração UNISAL. Ano 01. Número 01. Americana-Julho/Dezembro 2004. Disponível em: <http://www.am.unisal.br/pos/strictoadministracao/pdf/artigo-01-01-05.pdf>. Acesso em: 22/01/2007 


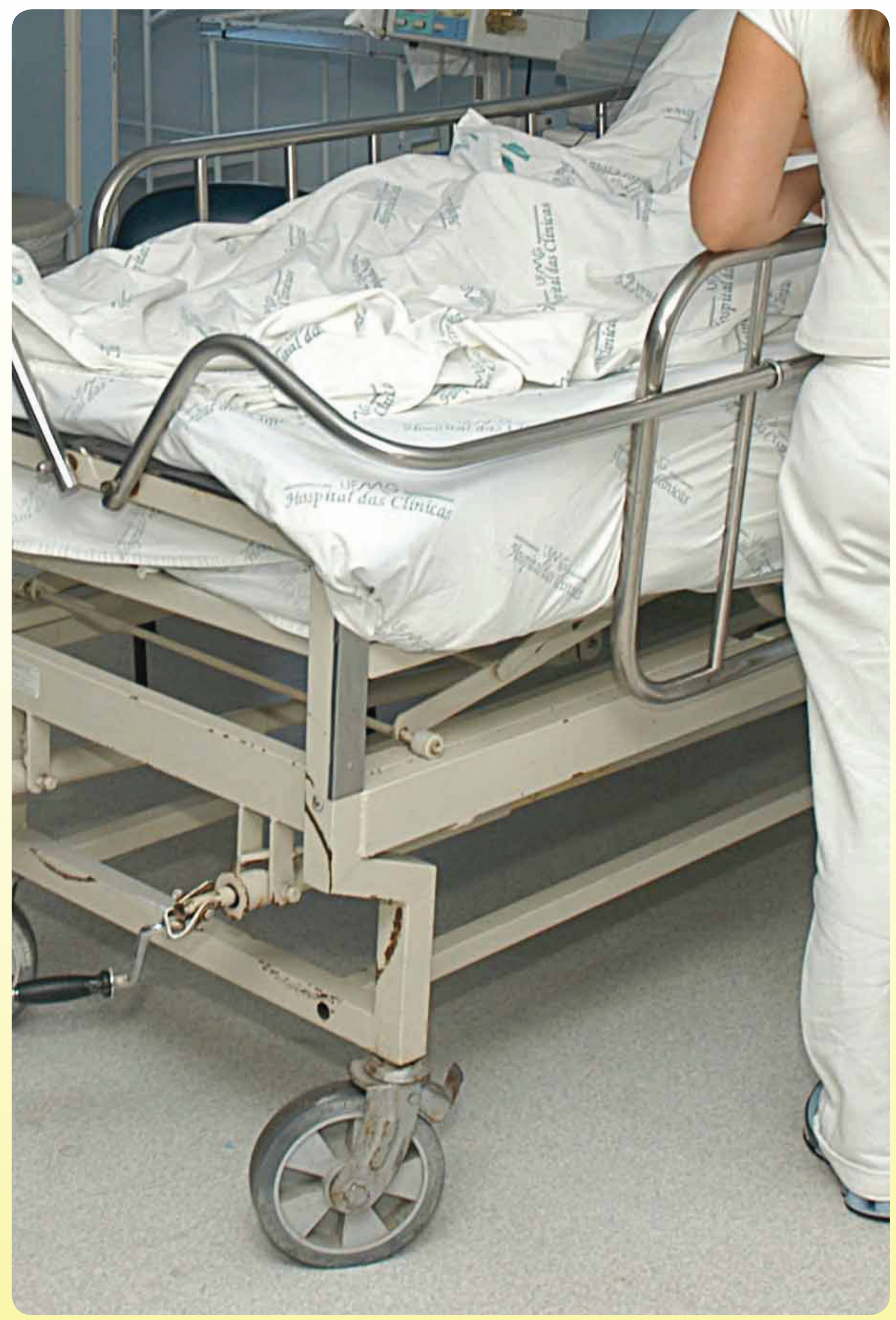

